\title{
The gene for a lectin-like protein is transcriptionally activated during sexual development, but is not essential for fruiting body formation in the filamentous fungus Sordaria macrospora Minou Nowrousian* and Patricia Cebula
}

\author{
Address: Lehrstuhl für Allgemeine und Molekulare Botanik, Ruhr-Universität Bochum, 44801 Bochum, Germany \\ Email: Minou Nowrousian* - minou.nowrousian@ruhr-uni-bochum.de; Patricia Cebula - patricia.cebula@gmx.de \\ * Corresponding author
}

Published: 03 November 2005

BMC Microbiology 2005, 5:64 doi:10.1186/147|-2180-5-64

This article is available from: http://www.biomedcentral.com/I47I-2/80/5/64

(c) 2005 Nowrousian and Cebula; licensee BioMed Central Ltd.

This is an Open Access article distributed under the terms of the Creative Commons Attribution License (http://creativecommons.org/licenses/by/2.0), which permits unrestricted use, distribution, and reproduction in any medium, provided the original work is properly cited.
Received: 10 September 2005

Accepted: 03 November 2005

\begin{abstract}
Background: The filamentous fungus Sordaria macrospora forms complex three-dimensional fruiting bodies called perithecia that protect the developing ascospores and ensure their proper discharge. In previous microarray analyses, several genes have been identified that are downregulated in sterile mutants compared to the wild type. Among these genes was tapl (transcript associated with perithecial development), a gene encoding a putative lectin homolog.

Results: Analysis of tap / transcript levels in the wild type under conditions allowing only vegetative growth compared to conditions that lead to fruiting body development showed that tap $I$ is not only downregulated in developmental mutants but is also upregulated in the wild type during fruiting body development. We have cloned and sequenced a $3.2 \mathrm{~kb}$ fragment of genomic DNA containing the tap I open reading frame and adjoining sequences. The genomic region comprising tapl is syntenic to its homologous region in the closely related filamentous fungus Neurospora crassa. To determine whether tapl is involved in fruiting body development in S. macrospora, a knockout construct was generated in which the tapl open reading frame was replaced by the hygromycin B resistance gene $h p h$ under the control of fungal regulatory regions. Transformation of the S. macrospora wild type with this construct resulted in a tap / deletion strain where tap / had been replaced by the $h p h$ cassette. The knockout strain displayed no phenotypic differences under conditions of vegetative growth and sexual development when compared to the wild type. Double mutants carrying the $\Delta t a p l$ allele in several developmental mutant backgrounds were phenotypically similar to the corresponding developmental mutant strains.
\end{abstract}

Conclusion: The tap I transcript is strongly upregulated during sexual development in $S$. macrospora; however, analysis of a tap / knockout strain shows that tap I is not essential for fruiting body formation in S. macrospora.

\section{Background}

Fruiting body formation in ascomycetes is a complex process leading to the formation of a number of specialized cell types from a comparatively undifferentiated veg- etative mycelium [1]. Recently, the molecular basis of this process has been investigated by forward and reverse genetics approaches, and a number of genes that are essential for fruiting body development have been identi- 


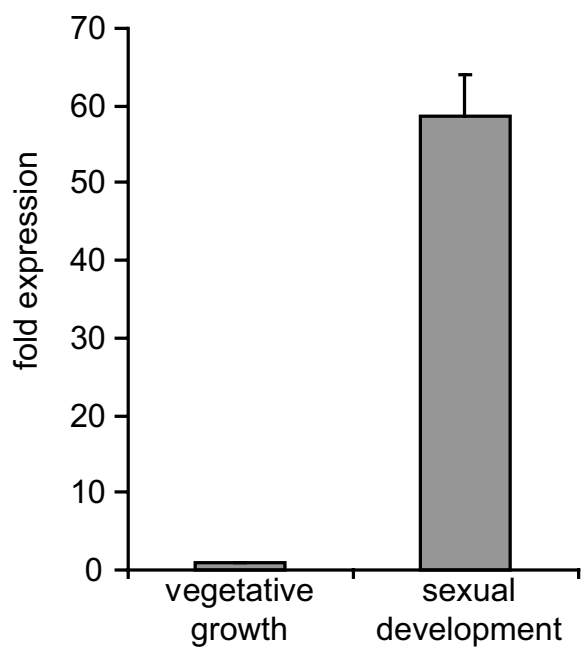

\section{Figure I}

Transcript levels of tap I are upregulated during sexual development. tap / transcript levels in the wild type under conditions of vegetative and sexual development were analyzed by real time PCR. For normalization, transcript levels of the SSUrRNAs were used as described previously [3]. Expression is given as fold induction (mean of two independent experiments, error bars indicate standard deviation) with transcript levels during vegetative growth set to I. Real time PCR results were tested for significance of differential expression at a level of $p=0.00 \mathrm{I}$ using REST [24].

fied. However, a coherent picture of fungal multicellular development has yet to emerge [2].

One avenue towards a deeper understanding of developmental processes is by functional genomics analyses, e.g. microarray studies. Such approaches can help to identify genes that are regulated differentially during fruiting body development and are therefore candidates for further functional analysis. In a previous study, we have performed microarray analyses of fruiting body development in S. macrospora [3]. This ascomycete is homothallic and produces fruiting bodies called perithecia within seven days under laboratory conditions. S. macrospora is a close relative of $N$. crassa, but in contrast to $N$. crassa, it does not produce any asexual spores. Therefore, changes of gene expression patterns during sexual development are not superimposed by changes related to asexual sporulation. We have previously analyzed gene expression in three developmental mutants of $S$. macrospora, and have identified a number of genes that are downregulated in the sterile mutants when compared with the wild type [3]. One of these genes is tap1 (transcript associated with perithecial development, formerly known as SMU5651 [3]). tap1 transcript levels are downregulated in the three developmental mutants pro1, pro11 and pro22 as well as in all three double mutants which led us to speculate that the gene might be involved in sexual development in $S$. macrospora. In addition to this intriguing expression pattern, the derived TAP1 amino acid sequence shows homology to lectins from other filamentous fungi with the highest similarity to lectins isolated from fruiting bodies of several basidiomycetes [3]. It has long been speculated that lectins play a role in fungal development; however, as no mutants in lectin-encoding genes from fruiting body-producing fungi have been analyzed to date, definite proof for this hypothesis is lacking [4,5]. The only known fungal lectin mutant is a strain of Arthrobotrys oligospora in which the lectin gene aol was deleted [6]. This mutant does not exhibit any phenotypical differences from the wild type under all conditions investigated, but as no sexual cycle is known for A. oligospora, the question whether aol might be involved in fruiting body development could not be addressed. Here, we present data on the expression of the S. macrospora tap1 gene as well as the characterization of a tap1 knockout strain to address the role of a putative lectin in fungal sexual development.

\section{Results \\ Expression of tapl during sexual development in S. macrospora}

Previously, we reported that tap 1 is downregulated in several developmental mutants when compared with the wild type [3]. In that analysis, all strains were grown under conditions that allowed sexual development, i.e. in floating culture. To investigate whether tap 1 expression in the wild type is associated with sexual development, we compared transcript levels of tap 1 in sexually developing mycelia with vegetative mycelia. For this purpose, the wild type was grown in floating or in submerged culture where it develops either fruiting bodies or vegetative mycelium, respectively. Analysis of tap 1 expression by quantitative real time PCR revealed that tap 1 transcript levels are upregulated nearly 60-fold in mycelia undergoing sexual development (Figure 1). These data further confirm that tap1 expression is linked with fruiting body development in S. macrospora.

\section{Sequence analysis of the S. macrospora tap I gene}

For previous expression analyses, part of the tap 1 open reading frame was amplified from $S$. macrospora genomic DNA by PCR [3]. For further characterization of tap1, we have isolated a tap1-containing cosmid clone from an indexed cosmid library of S. macrospora [7]. A $3.2 \mathrm{~kb}$ restriction fragment carrying the tap 1 open reading frame and adjacent regions was subcloned and sequenced. The fragment carries the uninterrupted tap 1 open reading frame of 429 bp encoding a predicted polypeptide of 143 


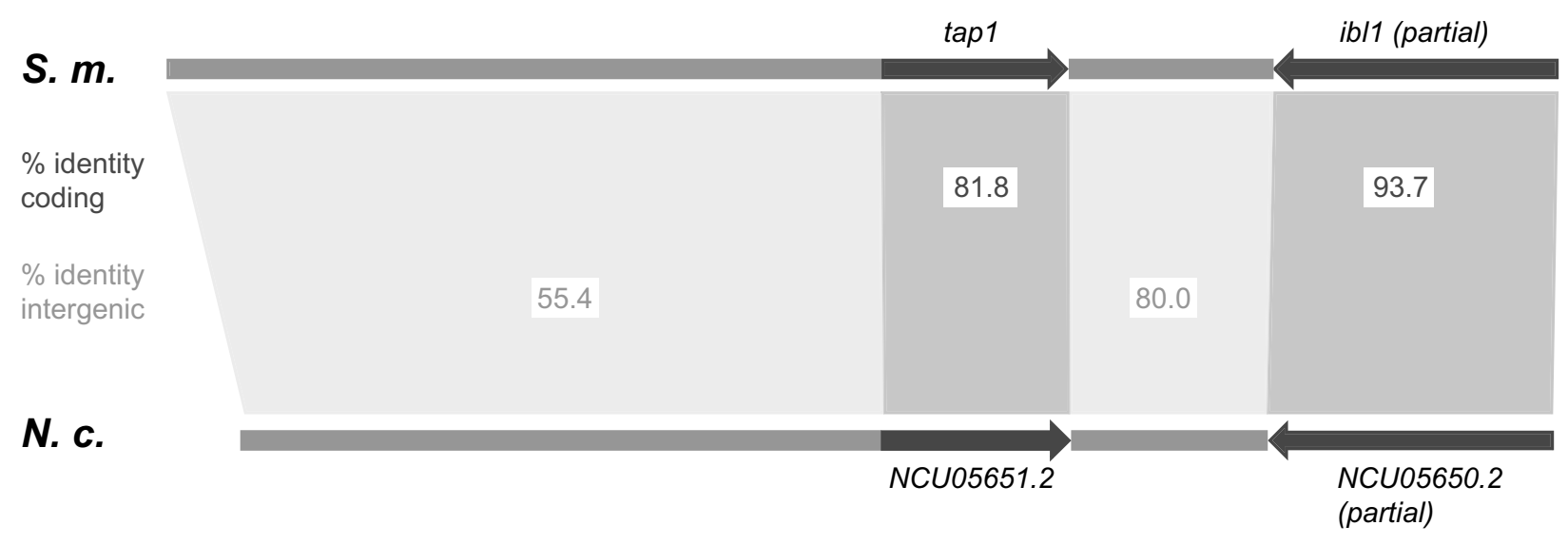

bp

11000

12000

13000

Figure 2

Synteny between S. macrospora (S.m.) and N. crassa (N.c.) in the genomic region containing tapl. Coding regions are given as dark gray and intergenic regions as light gray boxes. Nucleic acid identities between the $S$. macrospora open reading frames and their $N$. crassa orthologues as well as between the intergenic sequences are indicated.

amino acids. In addition, the fragment contains part of another open reading frame downstream of tap 1 that was named ibl1 (importin beta like 1) due to its similarity to an importin beta subunit. The sequenced region is syntenic to a region within the $\mathrm{N}$. crassa genome that contains the open reading frames NCU05651.2 and NCU05650.2 that are orthologs of tap1 and ibl1 respectively (Figure 2). In a previous comparison of 85 proteincoding genes of S. macrospora and N. crassa, the average nucleic acid identity within coding regions was found to be close to $90 \%$, and even non-coding regions of these two closely related Pyrenomycetes can be readily aligned [8]. These findings are further supported by our analysis of the genomic region containing tap1 (Figure 2).

The predicted TAP1 polypeptide was used for BLASTP [9] searches of the public databases, and a multiple alignment was constructed of TAP1 and several of its closest homologs from other fungi (Figure 3). The closest homolog is the predicted protein NCU05651.2 from N. crassa; however, interestingly, the second-best sequence identity is found in two lectins from the basidiomycetes Xerocomus chrysenteron and Paxillus involutus. Surprisingly, lectins and predicted lectin-like proteins from the ascomycetes Podospora anserina, Fusarium graminearum, and A. oligospora are less similar to the $S$. macrospora TAP1 even though these fungi are much more closely related to $S$. macrospora than the basidiomycetes (Figure 3). However, the (predicted) lectins from $P$. anserina, F. graminearum, and $A$. oligospora have a higher similarity compared to the basidiomycete lectins than TAP1 does (Figure 3). These findings might indicate that TAP1 and its corresponding $N$. crassa ortholog, even though being clearly members of this fungal lectin family, have evolved rather fast compared to other ascomycete homologs.

\section{Construction of a $\Delta$ tap I strain}

A construct for generating a tap1 deletion strain was generated. It consists of a hygromycin resistance cassette flanked by about $1 \mathrm{~kb}$ of sequences upstream and downstream of the tap1 open reading frame (Figure 4). The construct was used to transform the S. macrospora wild type, and transformants were screened for homologous recombination by PCR with oligonucleotide pairs $\mathrm{d} 1$ and $\mathrm{d} 2$ as well as $\mathrm{d} 3$ and $\mathrm{d} 4$ (Figure 4 ). These primer combinations yield amplicons only in the case of homologous recombination. Among 50 primary transformants, one transformant was found that displayed the expected PCR fragments (data not shown). Ascospores from this transformant were isolated to purify the putative knockout strain, and Southern blot analysis of nine ascospore isolates was performed with probes for tap 1 and the Hygromycin resistance gene hph. For two of the ascospore isolates, T2.35 and T2.41, results are shown in Figure 5. As expected, the tap1 probe hybridized to a $3.2 \mathrm{~kb}$ BglII fragment in genomic DNA of the wild type, whereas no hybridization signal was obtained with genomic DNA from the knockout strains T2.35 and T2.41. Conversely, the hph probe marked a $4.2 \mathrm{~kb}$ BglII fragment in the knockout strains and produced no signal with wild type 


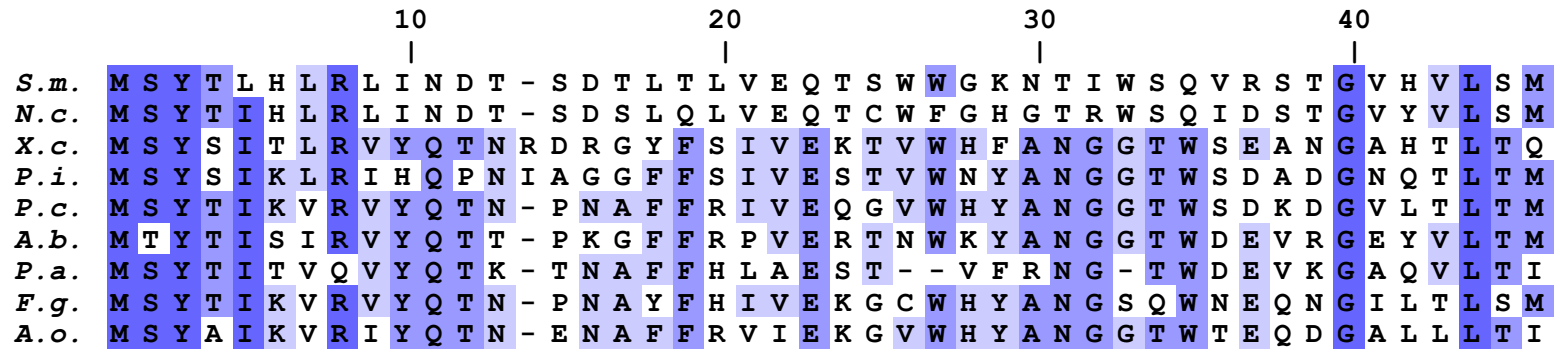

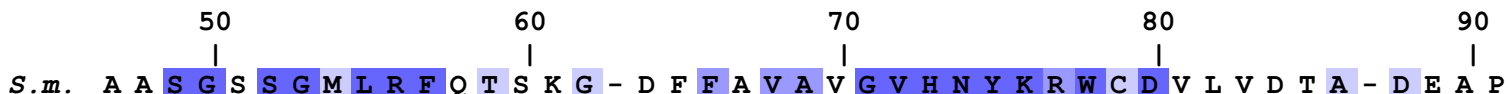

N.c. N N S G S S G M L R F Q N S K G G D Y F A V A V G V H N Y K R W C D I Q V D T A - D D A P I

X.c. G G S T S G V L R F L S - TKG ERI T V A V G V H N Y K R W C D V V T G L K - P D E T A

P.i. G G S G T S G T L R F M - D S G E R L I V A I G V H N Y K R W C D I A T G I A - P N A T G

P.c. G G S G T S G L R F M T E Q G K E A F F I A M G V H N Y K R W V D I V T G L A - N D V T C

A.b. G G S G T S S L R F V S S D T D E S F V A T F G V H N Y R W C D I V T N L T - N E $Q$ T A

P.a. G A S G T S G S L R F V A - D T G E N F I V T L G V H N Y K P W G D I V T K L D P A S Q T G

F.g. G G S G T S G M L R F K T E Q N K E G F F V A M G V H N Y K P W V D T I T G L G - D D I T C

A.O. G G S G T S I I R L Q T E $Q$ G K E A F F V A F G V H N Y K P W L D I I T G L G - D D I T G

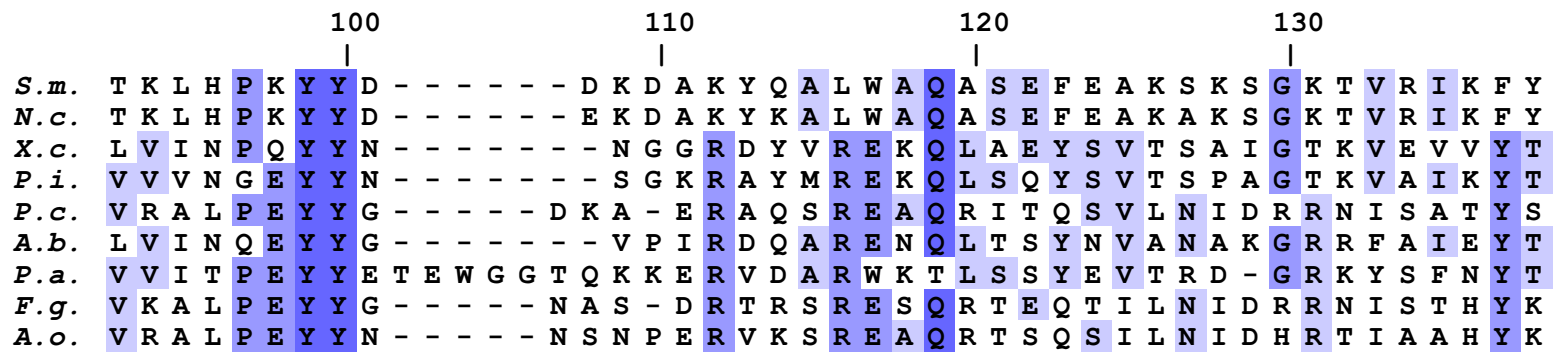

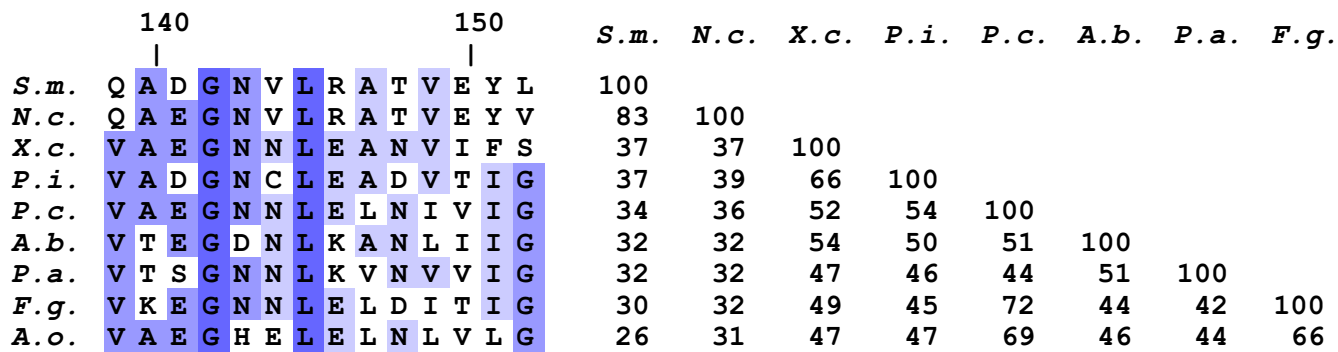

\section{Figure 3}

Multiple alignment of TAPI and lectins from filamentous fungi. The multiple alignment was created using CLUSTALX [25] with the following sequences: S.m., Sordaria. macrospora TAPI [this work, emb:CAH0368I.2]; N.c., Neurospora crassa NCU0565I.2 [ref:XP_325506.I]; X.c., Xerocomus chrysenteron lectin [gb:AAL73236.I]; P.i., Paxillus involutus LECA [gb:AAT91249.1]; P.c., Pleurotus cornucopiae PCL-F2 [dbj:BAB63923.I]; A.b., Agaricus bisporus ABL [sp:Q00022]; P.a., Podospora anserina Pa5D0092 [emb:CAD60779.I]; F.g., Fusarium graminearum FG07558.I [gb:EAA76455. I]; A.o., Arthrobotrys oligospora lectin [emb:CAA6578I. I]. Jalview was used to visualize the alignment [26]. Amino acid residues conserved in at least eight of the nine sequences are given in dark blue, residues conserved in at least six sequences in medium blue, and residues present in at least four sequences in light blue. At the end of the alignment, amino acid identity in \% is given for all sequences in pair-wise comparisons. 


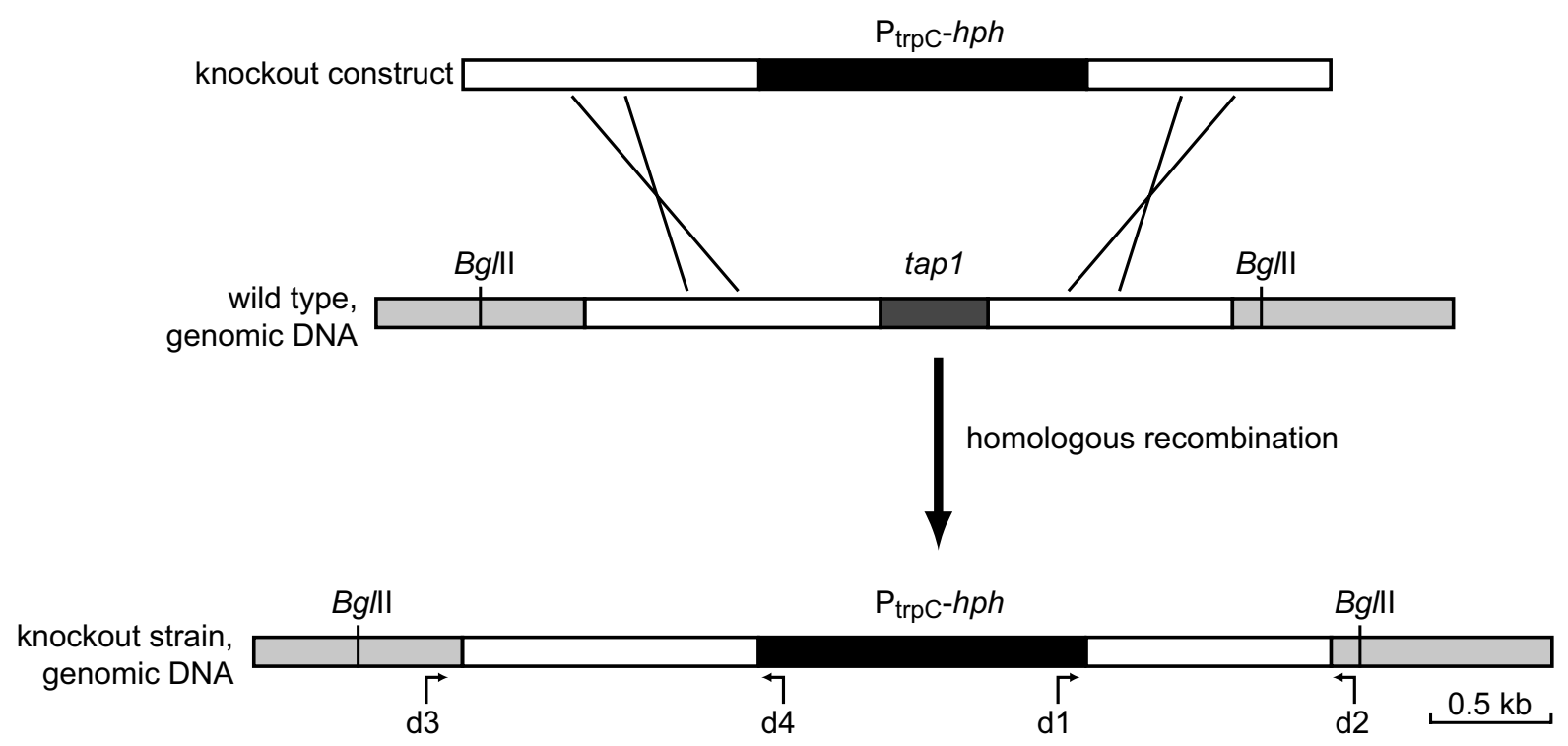

Figure 4

Strategy for the generation of a tapl knockout strain. The tap / open reading frame is shown in dark gray, flanking noncoding regions that are present in the knockout construct are given as white boxes, and adjoining regions not present in the knockout construct are shown in light gray. The Hygromycin B resistance cassette comprising the $h p h$ gene and the trpC promoter and 5' UTR is given in black. Positions of oligonucleotide primers $\mathrm{dl}$ to $\mathrm{d} 4$ are indicated. For further information see text.

DNA. The $4.2 \mathrm{~kb}$ fragment is of the size expected in a strain where homologous recombination has taken place (Figure 4). The $\Delta$ tap1 strains T2.35 and T2.41 were used for further analysis.

\section{Phenotypic characterization of the $\Delta t a p /$ strain in different genetic backgrounds}

The tap 1 deletion strains T2.35 and T2.41 were analyzed with respect to their phenotype during the sexual cycle. Surprisingly, both were completely wild type-like in all aspects of fruiting body development, i.e. both tap1 deletion strains produced mature perithecia in the same numbers and in the same amount of time as the wild type. Moreover, neither perithecial nor spore morphology were altered in any recognizable way (Figure 6). Fruiting body formation took place both in constant light as well as in constant darkness both on complete as well as minimal media, similar to that of the wild type. Ascospores from the knockout strains germinated readily and were black like wild type spores (Figure 6).

We then investigated whether there were any phenotypes unrelated to sexual development present in the mutant strains. Mycelial growth rates were determined as $24.7( \pm$
$1.7)$ and $24.8( \pm 1.4) \mathrm{mm}$ per day for $\Delta \operatorname{tap} 1$ and wild type respectively; thus, indicating that mycelial growth is not altered in the knockout strains. Also, renewal of growth and fruiting body development after incubation at $4^{\circ} \mathrm{C}$ or $37^{\circ} \mathrm{C}$, conditions that prevent growth and fruiting body formation, respectively, was not different in the mutants when compared to the wild type (data not shown). Also, wettability of mycelium and fruiting bodies was similar in wild type and mutants indicating that the hydrophobic coating of the mycelium was not altered in the knockout strains. As the lack of tap1 did not cause any discernable phenotype, we wondered whether tap1 might have a redundant function or whether its absence might be masked by the presence of other genes. We therefore crossed the $\Delta$ tap1 allele into strains bearing mutations in other developmental genes, namely pro1, pro11, pro22, and pro41. The pro1 mutant is defective in a gene encoding a transcription factor [10], the pro11 mutant lacks a functional WD40 repeat protein [11], and pro22 and pro41 are mutants non-allelic to tap1 or the other pro genes (Kück et al., unpublished data). tap1 transcript levels are downregulated in mutants pro1, pro11, pro22 [3], and pro41 (Nowrousian, unpublished data). Thus, we speculated whether a complete lack of tap1 would lead to 


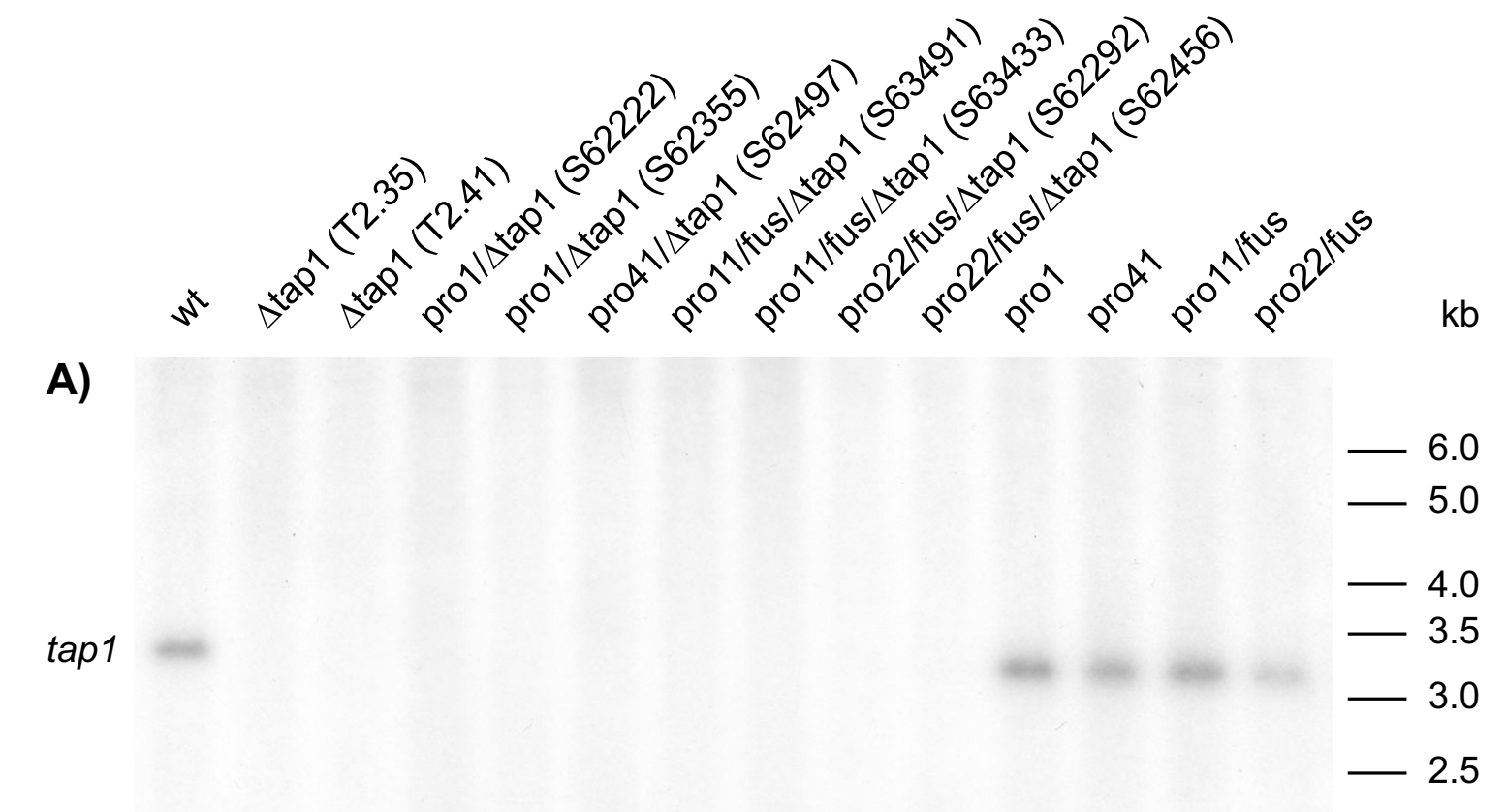

B)

\section{hph}

\section{Figure 5}

Southern blot analysis of $\Delta$ tap I strains. Genomic DNA from the wild type, from two different $\Delta$ tapl single spore isolates from the original knockout strain, and from double mutants of $\Delta$ tap I with prol, pro4 I, prol I, and prol I was hydrolyzed with $\mathrm{Bg} / \mathrm{ll}$, and after gel electrophoresis, the Southern blot was probed with radio-labeled DNA fragments containing the open reading frames of tapl (A) and hph (B), respectively. Marker sizes in kb are provided on the right. Numbers in brackets are strain numbers for single spore isolates.

a more pronounced developmental phenotype, especially as several other developmental genes are also downregulated in the pro mutants [3]. We obtained double mutants with strains pro1, pro11, pro22, and pro41 and verified the presence of the $\Delta t a p 1$ allele in the double mutants by Southern blot analysis (Figure 5). All double mutant strains were phenotypically similar to the respective single pro mutants in that they produced only protoperithecia and were therefore sterile. There were no differences in the number of protoperithecia produced by the double mutants when compared with the single mutants. Also, growth rates of the vegetative mycelium as well as overall 

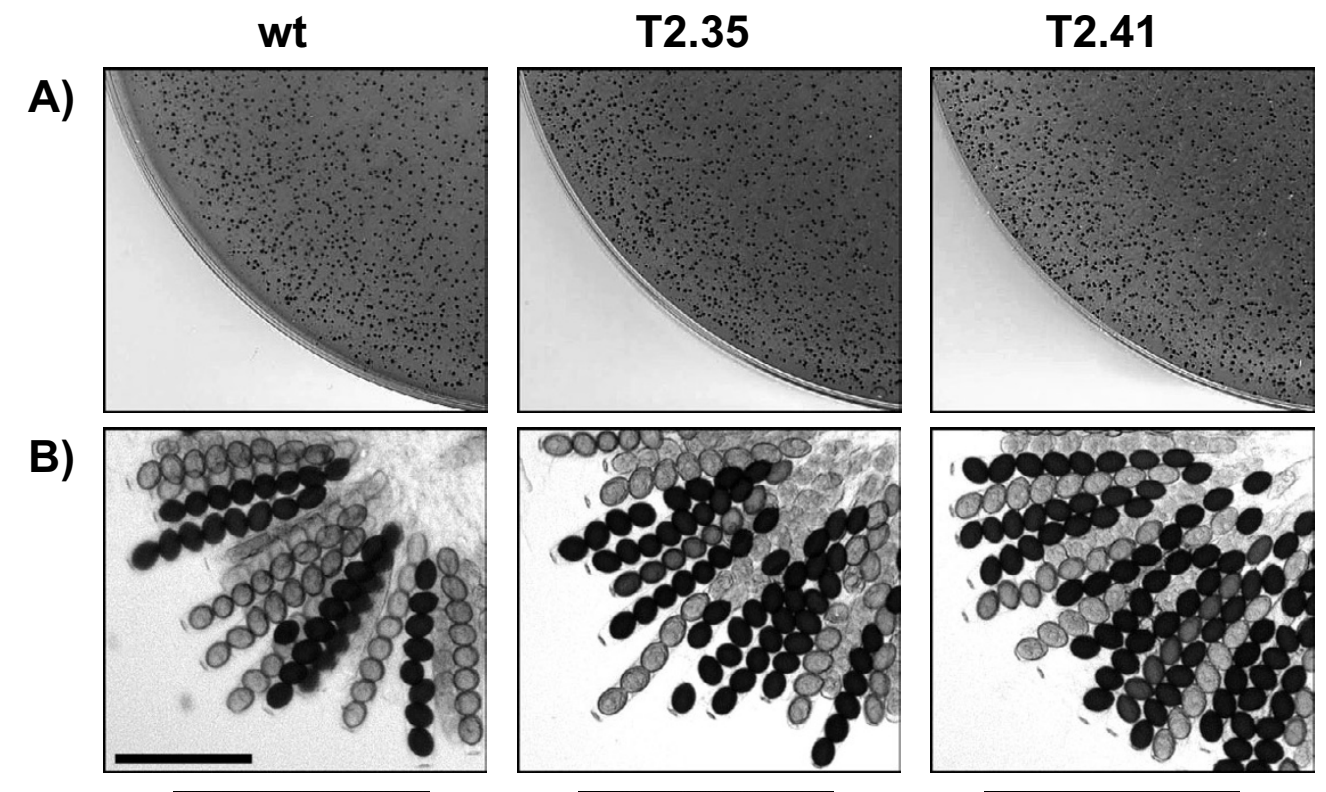

C)
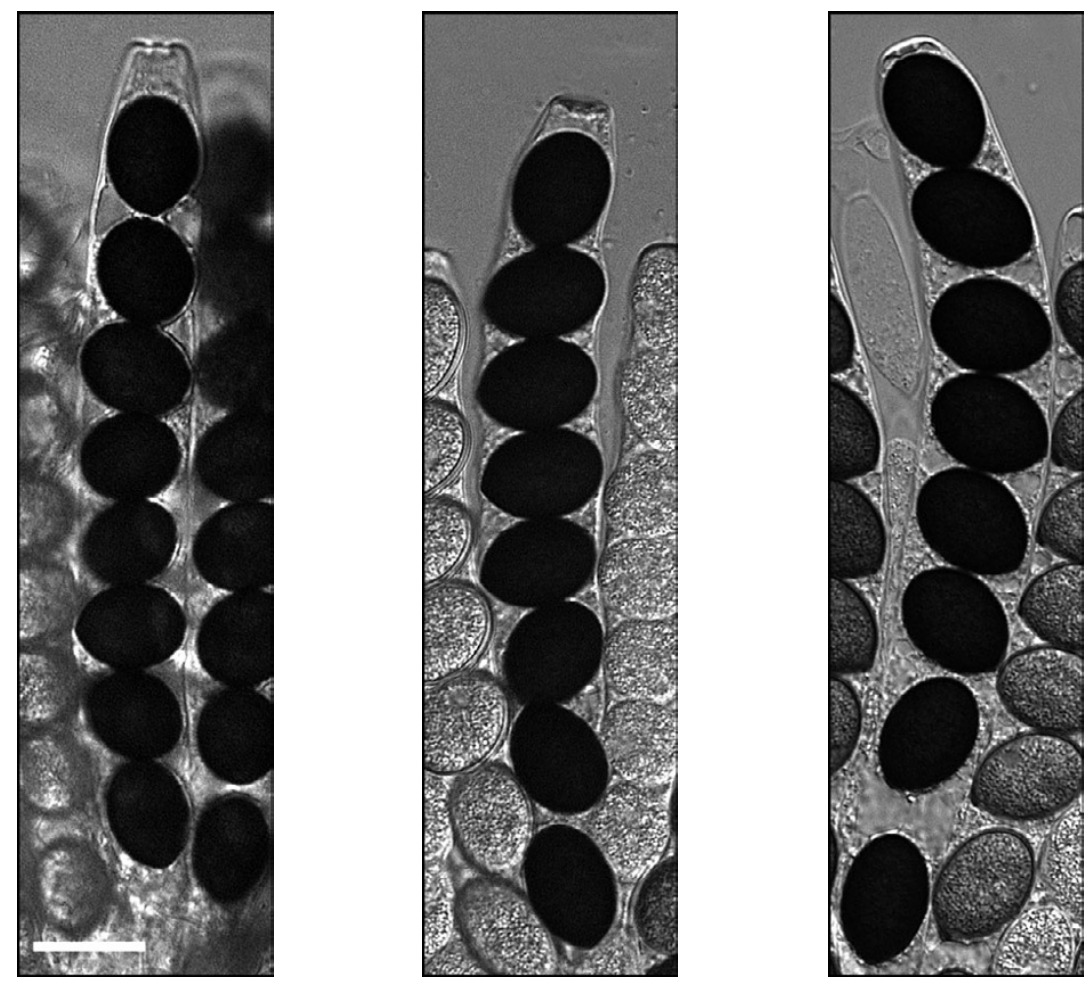

Figure 6

$\Delta$ tap I strains have a wild type-like phenotype. The wild type and $\Delta$ tapl single spore isolates T2.35 and T2.4I (as indicated above each column) were grown in Petri dishes on BMM solid medium [10, 2I] for $6 \mathrm{~d}(\mathrm{~A})$ or $7 \mathrm{~d}(\mathrm{~B}, \mathrm{C})$ at $25^{\circ} \mathrm{C}$ in constant light. A) Segments of petri dishes, black dots are individual fruiting bodies. B) Segments of ascus rosettes with mature (black-spored) and immature asci. Scale bar $100 \mu \mathrm{m}$. C) Mature asci. Scale bar $20 \mu \mathrm{m}$. 
morphological appearance were the same (data not shown). For the crosses of the tap1 knockout with the pro mutant strains and subsequent back-crosses, we analyzed a total of 17 full tetrads and 37 partial tetrads from crosses with different mutant strains and in all cases found the expected 1:1 segregation pattern for each of the single markers (hygromycin resistance and pro mutant phenotype, respectively). This is a further indication that deletion of tap1 does not interfere with sexual development, and also shows that the process of generating the $\Delta t a p 1$ allele did not introduce further mutations into the strains that would cause any different segregation patterns. Overall, no phenotype for $\Delta t a p 1$ was found in any of the genetic backgrounds investigated.

\section{Discussion}

The tap1-encoded polypeptide from S. macrospora has significant homology to lectins and lectin-like protein from other fungi (Figure 3). The highest degree of amino acid identity is found in comparison with lectins that were isolated from fruiting bodies of several basidiomycetes [1214]. Lectins are carbohydrate-binding proteins that are found in a variety of organisms $[4,5]$. On the basis of sequence homology, the $S$. macrospora TAP1 polypeptide can be included into a class of fungal lectins; however, whether it has lectin activity, i.e. whether it specifically binds carbohydrates, remains to be elucidated. Interestingly, TAP1 displays a greater sequence identity towards basidiomycete lectins than to lectins or putative lectins from ascomycetes with the (notable) exception of its closest homolog, the N. crassa protein NCU05651.2 (Figure 3). However, BLAST searches in the N. crassa genome [15] with the sequences of TAP1 as well as the lectin sequences from the other fungi used in our sequence comparisons yielded only NCU05651.2 as a significant result (data not shown). This finding indicates that the gene is present as a single copy in N. crassa, and that there is no other member of this lectin gene family present in the N. crassa genome. As $N$. crassa and $S$. macrospora are close relatives with highly syntenic genomes [8], it is likely that this is the case in S. macrospora as well. This observation is supported by the fact that only a single band in $S$. macrospora genomic DNA hybridizes with a tap1 probe (Figure 5). Thus, it seems that this particular class of fungal lectins has evolved faster in S. macrospora and N. crassa compared to other ascomycetes, for which it still retains more similarity with its basidiomycete relatives (Figure 3 ). Another class of fungal lectins has been found in the basidiomycete Coprinus cinereus. These lectins bind galactose and are therefore called galectins, and two galectins from C. cinereus are specifically expressed during different stages of fruiting body formation [16,17]. However, BLAST searches for galectin homologs in the $N$. crassa genome yielded no significant results (data not shown) indicating that no galectin-like proteins exist in N. crassa or that their sequences are too dissimilar to the C. cinereus sequences to be detected by sequence comparisons alone. Thus, the N. crassa NCU05651.2 gene and its S. macrospora ortholog tap1 are so far the only genes encoding putative lectins that have been identified by sequence analysis in these two ascomycetes.

In fungi, most lectins have been isolated from basidiomycetes, especially from mushroom fruiting bodies, and it has been speculated that they play a role in fruiting body development $[5,18,19]$. However, as no lectin mutant in a fruiting body-producing fungus has been characterized to date, this hypothesis has not been verified experimentally. Previous investigations and the results presented here show that tap 1 transcript levels are closely correlated with fruiting body development in S. macrospora; therefore, we decided to construct a tap1 knockout strain to analyze whether this putative lectin plays a role in fruiting body formation. A $\Delta$ tap 1 strain was generated by gene replacement, but the knockout strain has no discernable phenotype under all conditions investigated. This might indicate that $\operatorname{tap} 1$ has indeed no function in vegetative growth or sexual development of $S$. macrospora; however, the possibility that tap1 is needed under environmental conditions not tested in our experiments or that its function is redundant or that in the absence of tap1, another gene product can take its place, cannot be excluded. The latter effect is well known in other organisms, and it was, for example, tested in a large-scale analysis of yeast synthetic lethal interactions where it was found that genes involved in similar biological processes, but not necessarily in the same regulatory pathway, can buffer one another in single mutant backgrounds but show a phenotype in the double mutant strain [20]. To test whether any of the known developmental genes of $S$. macrospora show this kind of genetic interaction with tap1, we obtained double mutants of $\Delta \operatorname{tap} 1$ with strains bearing mutations in the developmental genes pro1, pro11, pro22, or pro41. However, all double mutant strains were phenotypically similar to the respective pro mutant strains. tap1 transcript levels are downregulated in the pro mutants, and our analysis demonstrates that even the complete loss of tap1 does not worsen the condition of the mutants. This leaves open the possibility that tap 1 is necessary in a different genetic background or under different environmental conditions.

With respect to fruiting body formation, our results show that the putative lectin-encoding gene tap 1 is not an essential gene for this developmental process. As mentioned previously, the only other known fungal lectin mutant is the aol mutant of the nematode-trapping fungus A. oligospora [6]. Similar to our findings, the aol mutant also has no phenotype under all conditions investigated. Since no sexual stages from A. oligospora are known, these obser- 
Table I: S. macrospora strains used in this study. All strains are single spore isolates and are kept in our laboratory collection. The fus allele that is present in some of the strains is a spore color marker (brown instead of black spores) but has no influence on growth or fertility.

\begin{tabular}{|c|c|c|}
\hline strain number & genotype & description \\
\hline S48977 & wild type & wild type \\
\hline T2.35 & $\Delta$ tapl & tapl deletion strain \\
\hline $\mathrm{T} 2.4 \mathrm{I}$ & $\Delta$ tapl & tapl deletion strain \\
\hline M887I & prol & sterile mutant [10] \\
\hline S24II7 & proll & sterile mutant $[\mathrm{II}]$ \\
\hline S22528 & pro22 & sterile mutant \\
\hline S46357 & pro4I & sterile mutant \\
\hline S62222 & $\Delta$ tapl, prol & sterile mutant \\
\hline S62355 & $\Delta$ tapl, prol & sterile mutant \\
\hline S62497 & $\Delta$ tapl, pro4l & sterile mutant \\
\hline S6349I & $\Delta$ tapl, proll, fus & sterile mutant \\
\hline S63433 & $\Delta$ tapl, proll, fus & sterile mutant \\
\hline S62292 & $\Delta$ tapl, pro22, fus & sterile mutant \\
\hline S62456 & $\Delta$ tapl, pro22, fus & sterile mutant \\
\hline
\end{tabular}

vations do not include fruiting body formation; however, vegetative growth, conidiation, and nematode-trapping were unchanged in the aol mutant strain [6]. Thus, possible functions of this class of lectins and lectin-like proteins in filamentous fungi remain enigmatic.

\section{Conclusion}

tap1 expression is strongly associated with sexual development in S. macrospora. An analysis of the tap1 gene and its surrounding genomic region revealed a high degree of sequence identity and overall synteny with the corresponding region in the genome of $N$. crassa. Sequence comparisons of TAP1 with lectins and lectin-like proteins from other fungi indicate that it is most closely related to lectins isolated from basidiomycete fruiting bodies. However, analysis of a tap 1 knockout strain shows that tap 1 is not essential for fruiting body formation nor vegetative growth in S. macrospora. This is the case for a $\Delta t a p 1$ allele in an otherwise wild type genetic background as well as in combination with mutations in several developmental genes. Whether tap1 has any function under growth conditions not investigated here, e.g. in a more natural setting, remains to be elucidated.

\section{Methods}

\section{Strains and growth conditions}

Details of the S. macrospora strains used in this study are provided in Table 1. Double mutants were obtained from crosses of single mutant strains. Double mutant genotypes were verified by crosses against single mutants. Unless stated otherwise, standard growth conditions and DNAmediated transformation were as previously described $[10,21]$. For analysis of growth velocity, $30 \mathrm{~cm}$ long race tubes were filled with $15 \mathrm{ml}$ of medium, inoculated at one end and the growth front was marked every $24 \mathrm{~h}$ for 7 consecutive days. For RNA extraction from cultures developing fruiting bodies, S. macrospora was grown at $25^{\circ} \mathrm{C}$ in constant light in floating culture as described [3]. For RNA extraction from vegetative mycelium, a mycelial plug of $0.7 \mathrm{~cm}$ in diameter from a Petri dish with liquid medium [3] was inoculated into an Erlenmeyer flask with $100 \mathrm{ml}$ of liquid medium and shaken at $130 \mathrm{rpm}$.

\section{RNA extraction and quantitative real time PCR}

Extraction of total RNA and quantitative real time PCR were performed as described previously [3] with the following modifications: reverse transcription was performed with $400 \mathrm{U}$ Superscript II (Invitrogen) and 0.33 mM dNTPs, and real time PCR was carried out in a DNA Engine Opticon 2 (MJ Research).

\section{Identification of a cosmid clone carrying tap I and analysis of the tapl gene}

An indexed S. macrospora cosmid library [7] was screened for tap1 by PCR with oligonucleotides SMU5651-1 (5' CATCAACGACACCTCCGACACCC) and SMU5651-2 (5' CATCGGCCTGATAGAACTTGATCC). For a first round of screening, pooled DNA from 48 cosmid clones was used as a template, DNA from clones from positive pools was then subpooled and used for the next round of screening. This led to the isolation of cosmid D3 from pool VI518614 that contains the tap 1 gene. A $3.2 \mathrm{~kb}$ BglII restriction fragment carrying tap1 was subcloned from cosmid D3 into pBluescript II/KS+ (Stratagene). The insert of the resulting vector PPC24 was sequenced at MWG Biotech or GATC Biotech AG [emb:AJ781427.2].

\section{Construction of a $\Delta$ tap l strain}

To create a tap1 knockout construct for homologous recombination in S. macrospora, flanking regions upstream and downstream of the tap 1 open reading frame were amplified by PCR from S. macrospora genomic DNA using oligonucleotides SMU5651-BamHI (5' AGGATCCGTGATTCTCATGCTGTGGAAGGAAGC) and SMU5651-NheI (5' AGCTAGCTTTGGCGGTTTGGTTGGGGGGTTGGT) for the upstream region and oligonucleotides SMU5651-ApaI (5' AGGGCCCGTACTCGTCAGTGGGAAAGTGGGTGG) and SMU5651-SacI (5' AGAGCTCTATGCACTTGCTCCTCAAGCGTCTC) for the downstream region, introducing restriction sites as indicated in the oligonucleotide names. Additionally, the hygromycin-resistance cassette consisting of the hph gene from Escherichia coli and the trpC promoter from Aspergillus nidulans was amplified from plasmid pCB1004 [22] using oligonucleotides Hph-ApaI (5' AGGGCCCTCAACGGAACCCTATTCCTTTGCCC) and Hph-NheI (5' AGCTAGCAACTGATATTGAAGGAGCATTTTTGG). All PCR fragments were subcloned in pDrive (Qiagen) resulting in pDriveA (upstream region), 
pDriveB (downstream region) and pDrivehph (hygromycin-resistance cassette). Sequences of inserts and orientation within the vector was verified by restriction analysis and sequencing (MWG Biotech AG). The PCR fragment containing the downstream region was obtained by ApaI/ SacI digestion of $\mathrm{pDriveB}$ and cloned into ApaI/SacI digested vector $\mathrm{pDriveA}$ resulting in plasmid pDriveAB. The hygromycin-resistance cassette was obtained by digesting plasmid pDrivehph with NheI and ApaI and was cloned into plasmid pDriveAB hydrolyzed with NheI and ApaI resulting in the knockout plasmid pABXY. For transformation of S. macrospora, plasmid pABXY was digested with BamHI and SacI and the knockout cassette was obtained by gel elution. The knockout cassette was transformed into the $\mathrm{S}$. macrospora wild type and primary transformants were screened for homologous integration by PCR. For this purpose, total DNA was prepared from the transformants according to [23], and PCR was performed with oligonucleotides d1 (5' CGATGGCTGTGTAGAAGTACTCGC) and d2 TGCCTCCTCCGAGGCTGATAACCT) for the downstream region or d3 (5' CGGTGGGTAAGGTATCTCTGATG) and d4 (5' CACCGCCTGGACGACTAAACCAA) for the upstream region (Figure 4).

\section{Authors' contributions}

$\mathrm{MN}$ designed the study, performed expression analyses and isolation of the cosmid clone as well as part of the characterization of the knockout strain and wrote the manuscript. PC cloned and sequenced the tap1 gene, made the knockout construct and the tap1 knockout strain and carried out part of the characterization of the knockout strain.

\section{Acknowledgements}

The authors would like to thank Swenja Ellßel and Susanne Schlewinski for excellent technical assistance and Prof. Dr. Ulrich Kück for his generous support that enabled us to carry out this study as part of project AI of research initiative SFB 480 funded by the Deutsche Forschungsgemeinschaft (DFG).

\section{References}

I. Bistis GN, Perkins DD, Read ND: Different cell types in Neurospora crassa. Fungal Genet Biol 2003, 50:17-19.

2. Pöggeler S, Nowrousian M, Kück U: Fruiting body development in ascomycetes. In The Mycota I Edited by: Kües U, Fischer R. Berlin, Heidelberg: Springer; 2005 in press.

3. Nowrousian M, Ringelberg C, Dunlap JC, Loros JJ, Kück U: Crossspecies microarray hybridization to identify developmentally regulated genes in the filamentous fungus Sordaria macrospora. Mol Genet Genomics 2005, 273: I37-149.

4. Wang $\mathrm{H}, \mathrm{Ng} \mathrm{TB}$, Ooi VEC: Lectins from mushrooms. Mycol Res 1998, 102:897-906.

5. Guillot J, Konska G: Lectins in higher fungi. Biochem Syst Ecol 1997 , 25:203-230.

6. Balogh J, Tunlid A, Rosen S: Deletion of a lectin gene does not affect the phenotype of the nematode-trapping fungus Arthrobotrys oligospora. Fungal Genet Biol 2003, 39: I28-135.

7. Pöggeler S, Nowrousian M, Jacobsen S, Kück U: An efficient procedure to isolate fungal genes from an indexed cosmid library. J Microbiol Meth 1997, 29:49-6I.
8. Nowrousian M, Würtz C, Pöggeler S, Kück U: Comparative sequence analysis of Sordaria macrospora and Neurospora crassa as a means to improve genome annotation. Fungal Genet Biol 2004, 4I:285-292.

9. Altschul SF, Madden TL, Schaffer AA, Zhang J, Zhang Z, Miller W, Lipman DJ: Gapped BLAST and PSI-BLAST: a new generation of protein database search programs. Nucleic Acids Res 1997, 25:3389-3402.

10. Masloff S, Pöggeler S, Kück U: The prol+ gene from Sordaria macrospora encodes a $\mathrm{C}_{6}$ zinc finger transcription factor required for fruiting body development. Genetics 1999, 152:191-199.

II. Pöggeler S, Kück U: A WD40 repeat protein regulates fungal cell differentiation and can be replaced functionally by the mammalian homologue striatin. Eukaryot Cell 2004, 3:232-240.

12. lijima N, Yoshino H, Ten LC, Ando A, Watanabe K, Nagata Y: Two genes encoding fruit body lectins of Pleurotus cornucopiae: sequence similarity with the lectin of a nematode-trapping fungus. Biosci Biotechnol Biochem 2002, 66:2083-2089.

13. Crenshaw R, Harper SN, Moyer M, Privalle LS: Isolation and characterization of a cDNA clone encoding a lectin gene from Agaricus bisporus. Plant Physiol 1995, 107:1465-1466.

14. Trigueros V, Lougarre A, Ali-Ahmed D, Rahbé Y, Guillot J, Chavant L, Fournier $D$, Paquereau L: Xerocomus chrysenteron lectin: identification of a new pesticidal protein. Biochim Biophys Acta 2003, 1621:2992-2298.

15. Galagan J, Calvo S, Borkovich K, Selker E, Read N, Jaffe D, FitzHugh W, Ma L, Smirnov S, Purcell S, et al:: The genome sequence of the filamentous fungus Neurospora crassa. Nature 2003, 422:859-868.

16. Boulianne RP, Liu Y, Aebi M, Lu BC, Kües U: Fruiting body development in Coprinus cinereus: regulated expression of two galectins secreted by a non-classical pathway. Microbiology 2000, 146: | $84 \mid-1853$.

17. Cooper DN, Boulianne RP, Charlton S, Farrell EM, Sucher A, Lu BC: Fungal galectins, sequence and specificity of two isolectins from Coprinus cinereus. J Biol Chem 1997, 272: I5I4-I52I.

18. Oguri S, Ando A, Nagata Y: A novel developmental stage-specific lectin of the basidiomycete Pleurotus cornucopiae. J Bacteriol 1996, 178:5692-5698.

19. Kaneko T, Oguri S, Kato S, Nagata Y: Developmental appearance of lectin during fruit body formation in Pleurotus cornucopiae. J Gen Appl Microbiol 1993, 39:93-100.

20. Tong AH, Lesage G, Bader GD, Ding H, Xu H, Xin X, Young J, Berriz GF, Brost RL, Chang M, et al: Global mapping of the yeast genetic interaction network. Science 2004, 303:808-813.

21. Nowrousian M, Masloff S, Pöggeler S, Kück U: Cell differentiation during sexual development of the fungus Sordaria macrospora requires ATP citrate lyase activity. Mol Cell Biol 1999, 19:450-460.

22. Carroll AM, Sweigard JA, Valent B: Improved vectors for selecting resistance to Hygromycin. Fungal Genet Newsl 1994, 4 I:22.

23. Lecellier G, Silar P: Rapid method for nucleic acid extraction from petri dish-grown mycelia. Curr Genet 1994, 25: I22-I 23.

24. Pfaffl MW, Horgan GW, Dempfle L: Relative expression software tool (REST) for group-wise comparison and statistical analysis of relative expression results in real-time PCR. Nucleic Acids Res 2002, 30:e36.

25. Thompson JD, Gibson TJ, Plewniak F, Jeanmougin F, Higgins DG: The ClustalX windows interface: flexible strategies for multiple sequence alignment aided by quality analysis tools. Nucleic Acids Res 1997, 24:4876-4882.

26. Clamp M, Cuff J, Searle SM, Barton GJ: The Jalview Java alignment editor. Bioinformatics 2004, I 2:426-427. 